\title{
Arena Function: A Framework for Computing Capacity Bounds in Wireless Networks
}

\author{
Alireza Keshavarz-Haddad, Shiraz Univ. Iran, and Rudolf Riedi, HES-SO Switzerland.
}

\begin{abstract}
Bounds on the capacity of wireless networks often rely on simplifying assumptions and are given in terms of coarse network parameters such as the number of nodes. While useful due to their simplicity such bounds can significantly overestimate the achievable capacity in real world situations, ignoring actual network topology and traffic patterns. The results of this paper improve such analytical results on network capacity in several ways. At the heart of our methodology lies the concept of transmission arenas which indicate the presence of active transmissions near any given location in the network. This novel space-based approach is well suited to untangle the interactions of simultaneous transmissions. Avoiding a graph-based model of the network it opens new avenues of studying capacities. For homogeneous networks we recover classical bounds. However, our methodology applies to arbitrary networks and can, thus, inform placing and activating of nodes also in the presence of clustering. Our method works with all classical channel models and dimensions. It provides bounds on the transport capacity which involve only high level knowledge of node locations, such as the length of Euclidean Minimum Spanning Tree. As an additional novelty we establish bounds on wireless unicast and multicast capacities.
\end{abstract}

Index Terms-Wireless Network Capacity, Transport Capacity, Multicast Transport Capacity, Transmission Arena, Euclidean Minimum Spanning Tree, Euclidean Steiner Tree, Multicast Capacity.

\section{INTRODUCTION}

$\mathbf{T}$ HERE has been a growing interest to understand the fundamental capacity limits of wireless networks [1]-[6]. Results on network capacity are not only important from a theoretical point of view, but they also provide guidelines for system and protocol design in wireless networks. Hitherto, most research on wireless network capacity has focused on the capacity of large homogeneous networks with unicast or multicast information flows b e $\mathrm{t}$ ween $\mathrm{u} \mathrm{n}$ i formly distributed randomly selected nodes [1], [3], [4], [6]-[13].

In this paper, we study arbitrary wireless networks with the goal of assessing how topology and traffic $p$ atterns i mpact the network capacity. At the heart of our method lies the concept of a transmission arena, a domain defined for e ach transmission which represents the part of the space which is affected by the transmission in a simple yet accurate manner. Doing so,

Alireza Keshavarz-Haddad is an Assistant Professor with the School of Electrical and Computer Engineering, Shiraz University, Shiraz, Iran (Email: alireza@alumni.rice.edu)

Rudolf Riedi is Professor of Mathematics with the Department of Information and Telecommunications at the School of Engineering and Architecture Fribourg, HES-SO University of Applied Sciences of Western Switzerland (Email: rudolf.riedi@hefr.ch). He held an appointment as Associate Research Professor with the Department of Statistics, School of Engineering, Rice University at the time of submission.

Manuscript received April 14, 2016; revised on December 14, 2016 and August 05, 2017. we avoid a graph-model of the network and its inaccuracies for modelling wireless channel and interferences as well as the complex graph theoretical problems it may lead to. With the concept of the arena we propose a completely different approach to studying capacities that opens new avenues for analysis.

As with graph-based approaches, the capacity bounds obtained using the arenas depend on the chosen channel model. We develop results for several well known wireless channel models used in [1], [14] in $d=1,2,3$-dimensional space.However, the theory developed is valid also for other channels models and for random or specific traffic patterns.

The effectiveness of the arenas in capturing topological information becomes apparent through a bound on the transport capacity in terms of a simple topological quantity, namely the length of the Euclidean Minimum Spanning Tree (EMST) of the network. As a special case of this result, one can recover the classical bound on the transport capacity of Gupta and Kumar [1], [15] using well known bounds on the length of the EMST. However, our bound using the EMST applies to arbitrary networks. In addition, we establish its tightness up to a logarithmic term in the number of the nodes of the network. As a consequence, our approach provides insight into design and operation of wireless networks. Placing or activating nodes such as to increase the length of the EMST will increase capacity.

The transport capacity of a wireless network is defined by considering unicast flows in the network. This quantity can provide an upper bound on the total throughput of unicast sessions, when the average distance between source-destination pairs of the unicast sessions are given. In this paper, we introduce a generalized version of transport capacity, called , multicast transport capacity. We establish an upper bound on the multicast transport capacity using the length of the EMST and show how to use this fact to obtain an upper bound on the total throughput of multicast sessions in a wireless network. We can show that the existing upper bounds on the multicast capacity [6], [9], [10] easily using this analytical method.

Arenas prove useful in various forms when studying multicast. Clearly, the topological constraints are more complex than for the unicast case, creating the need to introduce a more advanced tool called diffusion-span which allows us to derive capacity bounds for multicast flows. This is achieved by averaging the arena-bound appropriately over space and time. Note that the broadcast nature of wireless channels renders the analysis of network capacity for multicast flows much more involved as compared to the unicast case. Despite this complexity, our novel framework has the potential to strongly support future studies on the capacity of wireless networks for 
multicasting.

It should be noted that arenas provide bounds that are also sensitive to traffic patterns. To compute the throughput of simultaneous flows, e.g., we average the arena-bound over time at particular fixed points of the space. We demonstrate the effectiveness of the method by deriving an upper bound (cutset bound) for the maximum information flow rate between two parts of the network. We focus on two practical cases, one: where there is a space gap between the two parts and two: when the node density varies between two parts of the network.

The paper is organized as follows. In Section II we summarize existing works on network capacity. We explain basic notations and channel models in Section III. In Section IV we introduce the concept of transmission arenas and establish its relevant properties. In Section V we compute novel topologybased bounds for the transport capacity. In Section VI we address capacity bounds for multicast flows. In Section VII we provide cutset bounds for given data flows. We conclude the paper in Section VIII. Some of the more mathematical proofs are placed in the Appendix.

\section{RELATED WORK}

Gupta and Kumar [1] study the network capacity for unicast sessions in static wireless networks consisting of $n$ nodes distributed in a circle of area $A$ with wireless channel transmission rate $W$. They define the "transport capacity" of a wireless network with units of bit-meters per second as the maximum sum rate of the packets multiplied by the distance they travel between the source and the destination. Their main result says that the transport capacity of unicast sessions is $O(W \sqrt{A n})$ in an arbitrary network and it is $\Omega(W \sqrt{A n / \log (n)})$ in a random network where the nodes are placed uniformly. Here, we adopt the standard notation from complexity theory where $O($.$) ,$ $\Omega($.$) , and \Theta($.$) stand for asymptotic upper, lower, and tight$ bounds, respectively. As a result, if the capacity is shared between random sources and destinations in the network, the throughput capacity per node decreases as $O(W \sqrt{1 / n})$ (in random networks $\Omega(W \sqrt{1 / n \log (n)})$ ) when $n$ grows. The same authors also prove that if the nodes are distributed in a sphere with volume $V$ then the transport capacity is $O\left(W \sqrt[3]{V n^{2}}\right)$ [15]. Later, these results were generalized for another channel model based on Shannon's logarithmic function in [14].

Since the seminal work of Gupta and Kumar, network capacity has been studied using different approaches. By applying results from percolation theory, e.g., [4] introduces a scheme for random unicast source and destination pairs in a large random homogeneous network whichachieve $\Theta(W \sqrt{1 / n})$ per node capacity. Other authors compute different capacity bounds for wireless networks with multiple channels [16], [17], ultra-wide-band channel [18], directional antennas [19], hybrid wired backbone [20], and social network wireless links [21].

For wireless mobile networks, Grossglauser and Tse [2] show that per node capacity can be increased to $\Theta(W)$ if packet delay is left unbounded. They propose a mobilitybased routing method which uses at most two transmissions for transporting unicast packets. They consider a mobility model where the nodes move uniformly within a circular area; a mobile node close to the source receives the packet and moves in the entire network randomly and later delivers the packet when it is close enough to the destination. Many other efforts demonstrate the trade-off between the capacity and the delay in mobile networks with different mobility patterns and delay constraints (see [22] for references).

Note that the mentioned papers consider a regular type network topology (in random networks, the nodes are distributed homogeneously in the area) with symmetric traffic pattern (the traffic is distributed among the nodes in a uniform random fashion) for proving the achievability of the computed upper bounds on the network capacity. However, for a different network topology or traffic pattern the network capacity could become significantly smaller than these upper bounds. Indeed, in the analysis of [1], [4], [14] the effect of topology and traffic pattern are ignored and the computed upper bounds are only in terms of the number of nodes $(n)$ and the area of the network $(A)$. Also, there are some papers that study the network capacity for particular large scale heterogenous networks with non-uniform traffic [23]-[30].

However, the analytical results mentioned above can be applied only in wireless networks with special topology and traffic pattern. Introducing a new direction in network capacity research, the present paper goes well beyond this existing work, taking network topology and traffic pattern into account. Notably, this present work completes our previous conference paper [31] and provides more technical details and some new network capacity bounds.

It should also be mentioned that there exists work on the capacity of wireless networks for multicast and broadcast flows [3], [5], [6], [6], [9]-[11], [32]-[34]. These asymptotic capacity bounds are usually computed in random homogeneous networks with symmetric random traffic. Interestingly, the framework of the present paper can be generalized and applied for computing the capacity of arbitrary networks for multicasting and broadcasting. In fact, our results cover most of the traditional bounds on unicast, multicast and broadcast capacity.

Note that all the above mentioned papers as well as the present paper assume only simple wireless transmission and coding for the channel. If the nodes are allowed to use cooperative communication techniques and sophisticated multi-user coding then a per-node capacity of a higher order than that described above can be achieved [35]-[38]. A full discussion of these results is beyond the scope of this paper due to space constraints.

\section{Wireless Channel Models AND BASIC Notions}

In this section, we describe the models and notions used in this paper. We consider a wireless network consisting of $n$ wireless nodes in $d=1,2,3$-dimensional space $\left(\mathbb{R}^{d}\right)$. We denote the set of transmitter-receiver pairs of simultaneous direct transmissions active at time instant $\tau$ by

$$
Q:=\left\{\left(S_{1}, D_{1}\right),\left(S_{2}, D_{2}\right), \ldots,\left(S_{m}, D_{m}\right)\right\}
$$


Also, we denote the set of transmitters by $\mathcal{S}:=\left\{S_{1}, \ldots, S_{m}\right\}$. Note that in multi-hop networks these sets vary over time, but we usually consider one fixed but arbitrary time instant to analyze the network throughput. For simplicity of notation, the node symbols are used also to represent their locations. For example, $\left|S_{i}-D_{i}\right|$ is the Euclidean distance between the nodes $S_{i}$ and $D_{i}$ in $\mathbb{R}^{d}$. Notice that the nodes can be transmitting, receiving, or idle at different time instants. We use different notations for transmitters and receivers; the only reason for doing so is to improve clarity of formulas and the formulation of analytical results. Most existing papers have preferred to use the same notation for transmitter and receiver nodes.

\section{A. Wireless Channel Models}

This paper covers virtually all of the common channel models found in the literature on wireless network capacity, namely the following three groups of models. First, the Protocol Model defines a successful transmission based on the distance to the closest interfering transmitter [1], [7], [15]. This model is the simplest of the three and the easiest to analyze. Second, the Physical Model sets a threshold on the Signal to Interference plus Noise Ratio (SINR) of the received signal, declaring the transmission to be successful if the SINR is larger than a given threshold [1], [14], [15]. Third, the Generalized Physical Model determines the transmission rate in terms of the SINR [3], [4], [14] by using Shannon's capacity formula for a wireless channel with additive Gaussian white noise.

1) and 2): Protocol and Physical Model: In both, the Protocol and the Physical Model the assigned transmission rate from node $S_{i} \in \mathcal{S}$ to node $D_{i}$ is modelled as

$$
W_{i}= \begin{cases}W & \text { if the transmission is successful } \\ 0 & \text { if the tranmission is unsuccessful }\end{cases}
$$

where $W$ is the transmission rate.

What distinguishes the models are the conditions for a transmission to be modelled as successful. Current literature is mainly concerned with the following three different versions of the "Protocol Model" (see [1], [15]). Given the interference parameter $\Delta>0$ a transmission between $S_{i}$ and $D_{i}$ is modelled as successful if:

- (Protocol Model 1):

$\left|S_{k}-D_{i}\right| \geq(1+\Delta)\left|S_{k}-D_{k}\right|$ for all $S_{k} \in \mathcal{S} \backslash\left\{S_{i}\right\}$.

- (Protocol Model 2):

$\left|S_{k}-D_{i}\right| \geq(1+\Delta)\left|S_{i}-D_{i}\right|$ for all $S_{k} \in \mathcal{S} \backslash\left\{S_{i}\right\}$.

- (Protocol Model 3):

$\left|S_{k}-D_{i}\right| \geq(1+\Delta) r$ for all $S_{k} \in \mathcal{S} \backslash\left\{S_{i}\right\}$, and $\left|S_{i}-D_{i}\right| \leq r$

where the transmission range $r$ is an additional parameter.

Under the Physical Model a transmission is modelled as successful if

$$
\operatorname{SINR}=\frac{P_{i} G_{i i}}{N+\sum_{k \neq i, k \in \mathcal{S}} P_{k} G_{k i}} \geq \beta
$$

Here, $\beta$ is the SINR-threshold, $N$ represents the ambient noise, and $G_{k i}$ denotes the signal loss, meaning that $P_{k} G_{k i}$ is the receiving power at node $D_{i}$ from transmitter $S_{k}$. We assume a power-law decay for the signal loss of the form $G_{k i}=\mid S_{k}-$ $\left.D_{i}\right|^{-\alpha}$, where $\alpha>0$.
3) Generalized Physical Model: In this model all node pairs are able to communicate by direct transmission, however with a rate $W_{i}$ that depends on SINR as

$$
W_{i}=B \log _{2}\left(1+\frac{P_{i} G_{i i}}{B N_{0}+\sum_{k \neq i, k \in \mathcal{S}} P_{k} G_{k i}}\right)
$$

Here, $B$ is the bandwidth of the wireless channel and $N_{0} / 2$ is the noise spectral density. While this model assigns a more realistic transmission rate at large distances than the other two channel models, it also results in a singularity under the signal loss model $G_{i i}=\left|S_{i}-D_{i}\right|^{-\alpha}$ : according to (4) the receiving power and the rate are amplified to unrealistic levels if transmitter and receiver are placed very close to each other. The singularity can be easily addressed by upper bounding the received power at each node. Some papers have pointed out this issue [39], [40] and suggest a "bounded propagation model" for the rate. We do not study this version of the model in this paper, since its analysis can be performed in a straightforward way by using similar methods as put forward here for the two Physical Models.

\section{B. Transport Capacity}

The transport capacity is useful in order to study a set of unicast sessions among source-destination pairs $\mathcal{U}:=$ $\left\{\left(U_{1}, V_{1}\right), \ldots,\left(U_{m}, V_{m}\right)\right\}$. It can be defined as [1]:

$$
C_{T}(\mathcal{U}):=\max _{\text {unicast paths }} \sum_{k}\left|U_{k}-V_{k}\right| R_{k}
$$

where $R_{k}$ is the average throughput of the unicast session from $U_{k}$ to $V_{k}$. The maximum is taken over all possible multihop routes establishing the required connections between the sources and destinations. Note that the unit of transport capacity is "bit-meter per second" which is different from the unit of throughput capacity (bit per second). Interestingly, by computing the transport capacity of wireless network one can estimate the average throughput rate of the unicast sessions if the average distance of sender and receiver pairs is given.

A simple upper bound which actually does not depend on the set $\mathcal{U}$ is found by noting that for the routes achieving $C_{T}(\mathcal{U})$ there must be a time instance where the simultaneous direct transmissions which transport the information of the unicast sessions reach at least $C_{T}(\mathcal{U})$ [1]. Therefore,

$$
C_{T}(\mathcal{U}) \leq C_{T}=\max _{Q} \sum_{\left(S_{i}, D_{i}\right) \in Q}\left|S_{i}-D_{i}\right| W_{i}
$$

where the maximum is over all possible sets of simultaneous successful direct transmissions $Q$ in (1).

\section{Multicast Transport Capacity}

In this paper, we introduce multicast transport capacity as a generalized version of transport capacity that is defined for a set of multicast sessions instead of unicast sessions. Assume a given set of multicast sessions $\mathcal{M}:=\left\{\left(U_{1},\left\{V_{1}^{(1)}, \ldots, V_{1}^{\left(K_{1}\right)}\right\}\right), \ldots,\left(U_{m},\left\{V_{m}^{(1)}, \ldots, V_{m}^{\left(K_{m}\right)}\right\}\right)\right\}$, where $U_{i}$ and $\left\{V_{i}^{(1)}, \ldots, V_{i}^{\left(K_{i}\right)}\right\}$ represent the source and destinations of the $i$ th multicast session. We define the multicast 
transport capacity as

$$
C_{M T}(\mathcal{M}):=\max _{\text {multicast trees }} \sum_{k} L_{k} R_{k}
$$

where $L_{k}$ is the length of the Euclidean Steiner Tree (EST) that connects the source node $U_{i}$ to its destinations $\left\{V_{i}^{(1)}, \ldots, V_{i}^{\left(K_{i}\right)}\right\}$ and $R_{k}$ is the average throughput of this multicast session. The EST is a collection of line segments with three properties: (i) each line segment joins two nodes of the network and stands for the one-hop direct transmission between these nodes. (ii) Any pair of sender $U_{i}$ and receiver $V_{i}^{k}$ of $\mathcal{M}$ can be interconnected by following line segments of the EST. (iii) the EST has the minimum length among all collections that satisfy (i) and (ii). It may be shown that the line segments do not intersect each other.

We will present useful bounds on the multicast transport capacity which rely on the EST. More concretely, in order to compute our bounds, one will have to find the length of the EST first, a task which is known be an NP-hard problem in general. However, many efficient approximation solutions and tight bounds exist for the EST (to learn more see [41]). These bounds can then be translated into bounds on the multicast transport capacity.

Similar to (6), a simple upper bound on the multicast transport capacity is found as follows: for the routes achieving $C_{M T}(\mathcal{M})$ there must be a time instance where the simultaneous direct transmissions which transport the information of the multicast sessions, must reach at least $C_{M T}(\mathcal{M})$. Therefore,

$$
C_{M T}(\mathcal{M}) \leq C_{M T}=\max _{Q} \sum_{\left(S_{i},\left\{D_{i}^{(1)}, \ldots D_{i}^{\left(\kappa_{i}\right)}\right\}\right) \in Q} L_{i} W_{i}
$$

where the maximum is over all possible sets of simultaneous successful direct one-to-many transmissions $Q:=$ $\left\{\left(S_{1},\left\{D_{1}^{(1)}, \ldots, D_{1}^{\left(\kappa_{1}\right)}\right\}\right), \ldots,\left(S_{m},\left\{D_{m}^{(1)}, \ldots, D_{m}^{\left(\kappa_{m}\right)}\right\}\right)\right\}$ and where $L_{i}$ is the length of EST that connects the transmitter $S_{i}$ to its receivers $D_{i}^{(1)}, \ldots D_{i}^{\left(\kappa_{i}\right)}$ and $W_{i}$ is the transmission rate towards all these receivers.

Note that here we assume one-to-many transmissions with the same rate toward all receivers. If we allow multi-rate transmissions that provide higher rates to closer nodes, then a new weighted EST must be defined for the transmissions where each point has a weight proportional to its potential reception rate from the transmitter. A full discussion on the multi-rate case is, however, beyond the scope of this paper.

Based on the definitions, it is trivial to show that $C_{T} \leq C_{M T}$ in any wireless network. In the next section we will show that these two quantities possess the same upper bound and, moreover, that $C_{M T} / C_{T}=O(\log (n))$.

\section{ARENAS: A SPATIAL FRAMEWORK FOR SIMULTANEOUS TRANSMISSIONS}

The problem of finding the optimal configuration for simultaneous transmissions and therefore the capacity of a network requires complex and involved computations. In fact, this problem is NP-hard (see [42]) and there are no closedform expressions available for the capacity. Our method, on the other hand, provides an analytically tractable way to compute close bounds for the capacity. To untangle the mutual interference of simultaneous transmissions and to achieve analytically tractable yet asymptotically tight approximations, we move from the natural graph-based methodology to a space-based approach. In other words, rather than studying mutual restrictions and interference on the graph, we focus on restrictions regarding the proximity of ongoing transmissions as seen at any location $X$ of the space occupied by the network.

\section{A. Methodology of Our Space-based Approach at a simple example}

To explain our methodology let us consider a set of simultaneous successful transmissions under Protocol Model 3 in a planar network. It follows immediately from the definitions and the triangular inequality that the senders need to be at least at distance $\Delta r$ from each other. Much of the work existing today uses a graph-theoretical approach to deal with such restrictions. For our space-based approach consider an arbitrary point $X$ in the plane, not necessarily a sender or receiver. Consider the senders within distance $r$ from $X$; as noted the discs of radius $\Delta r / 2$ around these senders must be disjoint, yet these discs are contained in the disc of radius $r+\Delta r / 2$ around $X$. Thus, there can be at the most $M:=(r+\Delta r / 2)^{2} /(\Delta r / 2)^{2}=(1+2 / \Delta)^{2}$ successful senders which include $X$ in their radio range.

Intuitively, this tells us that under the Protocol Model 3 the rate of information which can be transmitted in the vicinity of any arbitrary point $X$ is bounded by the constant $M \cdot W$. In other words, the "local capacity" of the network or "packing density" of senders is bounded everywhere by $M \cdot W$. This packing reminds one of tiling in cellular networks but is different. Denoting by $A_{i}$ the radio range of sender $S_{i}$ this fact can be expressed neatly as

$$
\sum_{i \in Q: X \in A_{i}} W_{i}=\sum_{i \in Q} W_{i} \cdot \mathbb{I}_{A_{i}}(X) \leq(1+2 / \Delta)^{2} \cdot W
$$

Here, $\mathbb{I}_{A_{i}}(X)$ is an indicator function that is equal to 1 if $X$ is located in the set $A_{i}$, and 0 otherwise.

\section{B. Unicast Transmission Arena: the Concept in General}

To generalize the example section IV-A, set

$$
W_{o}:= \begin{cases}W & \text { Protocol and Physical Models, } \\ B & \text { Generalized Physical Model. }\end{cases}
$$

in units of bit-per-second and introduce the arena-rate function

$$
\phi_{i}(X):=W_{i} \cdot \mathbb{I}_{A_{i}}(X)
$$

The set $A_{i}$ is called transmission arena, or arena for short, and will be chosen for each channel model such as to represent the transmission of rate $W_{i}$ (2) resp. (4) over the distance from sender $S_{i}$ to receiver $D_{i}$, as well as the effect of this transmission on others. The arena is allowed to depend on $S_{i}$ and $D_{i}$ alone. An illustrative example is given in IV-A. 


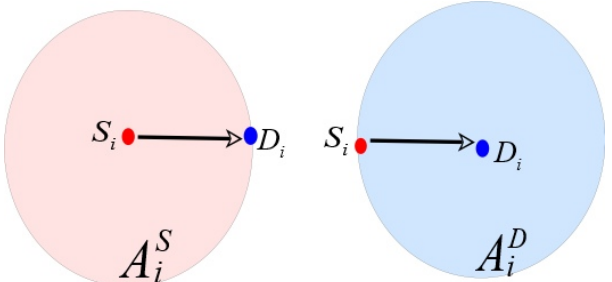

Fig. 1. The disturbance area $A_{i}^{S}$ and the protection area $A_{i}^{D}$ of a transmitterreceiver pair $\left(S_{i}, D_{i}\right)$ each form arenas for most channel models (see Lemma 4.1 and Lemma 4.2).

We define ${ }^{1}$ an arena-bound $M$ to be any positive number such that for any fixed time instance and any point $X \in \mathbb{R}^{d}$

$$
\sum_{i \in Q} \phi_{i}(X)=\sum_{i \in Q} W_{i} \cdot \mathbb{I}_{A_{i}}(X) \leq M \cdot W_{o}
$$

The arena-bound $M$ is allowed to depend only on the parameters of the channel model and the number of nodes: in particular, it may depend on $\# Q$ but not on node location or traffic patterns.

Note, the arena-rate function does not approximate the signal strength at $X$ but rather provides a weighted indication of the presence of transmission $i$ nearby $X$. Indeed, the potential of arenas lies less in capturing the relation between senders but rather in providing a spatial framework which compactly captures the interactions between simultaneous direct transmissions via (11) and their impact on any location $X$. Moreover, arenas are free of detailed information on topology or traffic patterns, and, most importantly, allow the use of integration and other analytical tools.

\section{Arenas for the Classical Channel Models}

An arena can be thought of as representing to a fair degree the interference caused at the sender, respectively the low noise level required at the receiver. Natural choices are:

$$
\begin{aligned}
\text { disturbance area } & A_{i}^{S}:=\left\{X:\left|X-S_{i}\right| \leq l_{i}\right\} \\
\text { protection area } & A_{i}^{D}:=\left\{X:\left|X-D_{i}\right| \leq l_{i}\right\}
\end{aligned}
$$

where $l_{i}=\left|S_{i}-D_{i}\right|$ (see Fig. 1). Extending the argumentation of the illustrative example in IV-A, we are able to establish arena-bounds for the appropriate choices of an arena under all models of Section III as stated in Lemma 4.1. Its proof is in the Appendix.

Lemma 4.1: Choose one of the following sets as transmission arena under the indicated channel models:

$$
A_{i}:= \begin{cases}A_{i}^{S} & \text { Protocol Model 1,3, Physical Model } \\ A_{i}^{D} & \text { Protocol Model 2,3, Physical Model } .\end{cases}
$$

\footnotetext{
1 More generally we could allow an arena-rate function to be any nonnegative function $\phi_{i}$ and define the arena-bound M to satisfy $\sum_{i \in Q} \phi_{i}(X) \leq$ $M \cdot W_{o}$. In this case, we would set the arena to be the domain of $\phi_{i}$. This level of generality can be used for various channel models to improve the bounds, but it is beyond the scope of this paper.
}

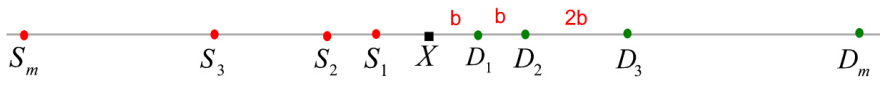

Fig. 2. If we set $P_{k}=k !\left(\beta 2^{\alpha}\right)^{k} P_{\min }$ and $l_{k}=2^{k} \epsilon$ where $\epsilon$ is a small constant, then all transmissions will be successful. Consequently, $\sum_{k} \phi_{k}(X)=$ $m W$ for all $X \in\left[S_{1}, D_{1}\right]$.

Then, the corresponding arena-bounds can be chosen independently of the number of sources as

$$
M:= \begin{cases}1 & \text { for } \Delta>2, \text { any Protocol Model, } \\ \left\lceil\frac{(4+2 \Delta)^{d}}{\Delta^{2 d}}-1\right\rceil & \text { for } \Delta \leq 2, \text { Protocol Models 1, 2, } \\ \left\lceil\frac{(2+\Delta)^{d}}{\Delta^{d}}-1\right\rceil & \text { for } \Delta \leq 2, \text { Protocol Model 3, } \\ \left\lceil\frac{3^{\alpha} P_{\max }}{\beta P_{\min }}\right\rceil & \text { for Physical Model. }\end{cases}
$$

For the Physical Model, we assume that there are two constants $P_{\max }$ and $P_{\min }$ which bound the transmission power of any node at any time from above and below.

Note that the above arena-bounds (15) depend only on the parameters of the channel model; in particular, they do not depend on the number of nodes.

To this end, the special assumption for the Physical Model guarantees that the ratio of the power of any two senders is bounded by $P_{\max } / P_{\min }$ independently of the number of senders. Fig. 2 shows a topology and set of simultaneous successful transmission such that the best arena-bound at point $X$ grows linearly with the number of nodes. Note that the maximal power grows here with the number of nodes.

However, such bounds are not tight in the sense of V-B and we prefer arena-bounds which are constant or at most logarithmic in the number of nodes such as in the next lemma.

Lemma 4.2: Under the Generalized Physical Model, for both $A_{i}^{S}$ and $A_{i}^{D}$ we may chose the arena-bound

$$
M:=\left(\frac{\max _{i}\left(W_{i}\right)}{B}+\frac{3^{\alpha} P_{\max } \log _{2}(e \cdot \# Q)}{P_{\min }}\right) .
$$

Alternatively, (11) holds also for

$$
M^{\prime}:=2 \log _{2}(e) \cdot \frac{P_{\max }}{P_{\min }}\left(\frac{3 l_{\max }}{l_{\min }}\right)^{\alpha}
$$

which depends on the network topology via $l_{\max }$ and $l_{\min }$, the maximum distance and minimum distance between transmitter and receiver pairs in $Q$. The constants Pmax and Pmin are as in Lemma 4.1.

\section{One-to-Many Arena-rate function}

Consider a wireless channel with a transmitter $S_{i}$ and a set of receivers $D_{i}^{(1)}, D_{i}^{(2)}, \ldots, D_{i}^{(m)}$. In analogy to (10) we define the one-to-many arena-rate function of this transmission to be

$$
\phi_{i}(X)=\max _{k=1, \ldots, m} \phi_{i}^{(k)}(X)
$$

where $\phi_{i}^{(k)}(X)$ is an arena-rate function of the transmission $S_{i}$ to $D_{i}^{(k)}$. This means that the one-to-many arena becomes the union of the arenas of successful transmissions from the transmitter to the receivers (see Fig. 3). It is essential to note: 

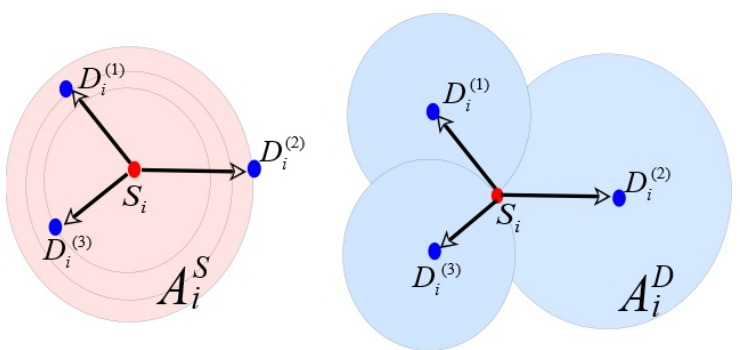

Fig. 3. The disturbance area $A_{i}^{S}$ of a transmitter and the protection area $A_{i}^{D}$ of a receiver form arenas for a one-to-many transmission $\left(S_{i},\left\{D_{i}^{(1)}, D_{i}^{(2)}, D_{i}^{(3)}\right\}\right)$.

Lemma 4.3: The arena-bound $M$ of these one-to-many arena-rate functions can be chosen as in the unicast case (see Lemmas 4.1 and 4.2).

For a proof, note that the one-to-many arena-rate function $\phi_{i}(X)$ at point $X$ equals the arena-rate function of one of the receivers, say $D_{i}^{\left(k_{i}\right)}$. Thus, we have $\sum_{i} \phi_{i}(X)=\sum_{i} \phi_{i}^{\left(k_{i}\right)}(X)$. Denoting by $Q^{\prime}$ the set of unicast pairs $\left(S_{i}, D_{i}^{\left(k_{i}\right)}\right)$ we have $\# Q=\# Q^{\prime}$ and we may use the same arena-bounds as in the unicast case.

\section{BOUNDS ON THE TRANSPORT CAPACITY}

In this section, we compute novel transport capacity bounds which are sensitive to network topology and hold for arbitrary traffic patterns. Using the concept of transmission arenas we are able to understand and quantify the impact of more detailed spatial information on wireless network capacity. This leads us to insights into design and operation of wireless networks: Placing or activating nodes such as to increase the length of the EMST will increase capacity (see below). Our computed upper bounds are also valid for multicast transport capacity that will be used in the following section to derive upper bounds on the multicast capacity.

\section{A. Upper Bound on $C_{T}$}

In this section we develop upper bounds for the transport capacity of an arbitrary wireless network in terms of the length of its Euclidean Minimum Spanning Tree (EMST). An EMST is a tree formed by the network nodes where the weights of the edges are the Euclidean distances of the nodes such that the total weight of the tree is minimal.

Theorem 5.1: Let $M$ be the arena-bound of the underlying channel model (see (15) and (16)). Then, the transport capacity of an arbitrary wireless network is bounded as

$$
C_{T} \leq M \cdot W_{o} \cdot L_{\mathrm{EMST}}
$$

where $L_{\mathrm{EMST}}$ is the length of EMST of the network.

Proof of Theorem 5.1: First, we establish a useful simple property in the following lemma. The claim follows easily from the fact that the portion of the curve $\Gamma$ located inside $A_{i}^{S}$ has a length of at least $l_{i}$. Similar for $A_{i}^{D}$.
Lemma 5.2: Consider a continuous curve $\Gamma$ that connects $S_{i}$ to $D_{i}$. Then, for both, $A_{i}=A_{i}^{S}$ and $A_{i}=A_{i}^{D}$, we have

$$
l_{i} W_{i} \leq \int_{\Gamma} \phi_{i}(X) d \ell
$$

Now, consider an arbitrary set of transmitter and receiver pairs $Q$ at a given time instant $\tau$ (see Section III-B). Denote the EMST curve by $\Gamma_{\text {EMST. }}$. Clearly, for every transmitter and receiver pair $\left(S_{i}, D_{i}\right)$, there exists a path in the EMST which connects the transmitter $S_{i}$ to receiver $D_{i}$. The proof of theorem 5.1 is completed by using lemma 5.2 as follows

$$
\begin{aligned}
\sum_{i \in Q} l_{i} W_{i} & \leq \sum_{i \in Q} \int_{\Gamma_{\mathrm{EMST}}} \phi_{i}(X) d \ell=\int_{\Gamma_{\mathrm{EMST}}} \sum_{i \in Q} \phi_{i}(X) d \ell \\
& \leq \int_{\Gamma_{\mathrm{EMST}}} M W_{o} d \ell=M W_{o} L_{\mathrm{EMST}}
\end{aligned}
$$

Note that theorem 5.1 applies to any channel model with an arena-rate function such that (20) holds and an arena-bound $M$ exists. In addition, theorem 5.1 applies to any network. When the nodes are distributed homogenously in the network the upper-bound (19) will become similar to the bounds in current literature. The well-known bounds of [1], [14], [15], e.g., are special cases of theorem 5.1. When the distribution of the nodes is nonhomogeneous most bounds existing today cannot be applied or are larger than (19). Some examples are given in [31].

Corollary 5.3: Let $M$ be the arena-bound of the underlying channel model. Assume that the network nodes are located in a d-dimensional cube with volume $V$. Then, the transport capacity is bounded as

$$
C_{T} \leq M \cdot W_{o} \cdot K_{d} \sqrt[d]{V n^{d-1}}
$$

where $K_{d}=1,3,6$ in $d=1,2,3$ dimensional space.

\section{B. On the Tightness of the Upper Bound on $C_{T}$}

As we establish next, the length of the EMST is indeed a key quantity since the bound of Theorem 5.1 differs typically from the maximum achievable transport capacity of the network by at most a factor of $O(\log (n))$.

Theorem 5.4: The following holds under all channel models of Section III except Protocol Model 3, and under assumption that for the Physical models $\alpha>d$ (see (3) and (4)).

Assume a well-connected ${ }^{2}$ wireless network with EMST size $L_{\mathrm{EMST}}$ is given. Then, there exists a traffic pattern in the network with transport capacity of $C_{T}$ such that

$$
C_{T} \geq K_{1} \cdot W_{o} \cdot L_{\mathrm{EMST}} / K_{2}
$$

where $K_{1}$ is a constant number, also $K_{2}=1$ if $d=1$ and $K_{2}=\log (n)$ if $d=2,3$. Recall that $d$ denotes the dimension of the network as defined at the beginning of Section III.

Note that the bound of Theorem 5.4 cannot be improved more than a constant if the length of EMST is the only

\footnotetext{
${ }^{2}$ To avoid pathologies we call a network well-connected, if there exists constants $0<\epsilon_{1}, \epsilon_{2}$ such that for every arbitrary two nodes there are a path, and a time scheduling for transporting data at rate $\epsilon_{1} W_{o}$ along the path with transmission power $\epsilon_{2} P_{\max }$.
} 
topology information of the network. A pathological example has been shown in [31]. Also, here we point out that tight upper and lower bounds can be derived for Protocol model 3 based on the Maximum Independent Set (MIS) of the network graph. These bounds have been presented earlier [31].

\section{Upper bound on $C_{M T}$}

Here, we develop an upper bound on the multicast transport capacity and use this bound to derive novel upper bounds on the multicast capacity of wireless networks under various topology and traffic patterns.

Theorem 5.5: Assume Protocol Model 2 or 3 or the Physical Model for wireless channel. Let $M$ be the arena-bound of the underlying channel model. Then, the multicast transport capacity of an arbitrary network is bounded as

$$
C_{M T} \leq M \cdot W_{o} \cdot L_{\mathrm{EMST}}
$$

where $L_{\mathrm{EMST}}$ is the length of EMST of the network.

Similar to Theorem 5.1, we establish first a Lemma.

Lemma 5.6: Consider a continuous curve $\Gamma$ that connects $S_{i}$ to $D_{i}^{(1)}, D_{i}^{(2)}, \ldots, D_{i}^{(m)}$. Then, for $A_{i}^{D}=\bigcup_{j=1}^{m} A_{i}^{D_{i}^{(j)}}$, we have

$$
L_{i} W_{i} \leq \int_{\Gamma} \phi_{i}(X) d \ell
$$

where $L_{i}$ is the length of Euclidean Steiner Tree (EST) that connects the transmitter $S_{i}$ to its receivers $D_{i}^{(1)}, D_{i}^{(2)}, \ldots, D_{i}^{(m)}$.

The lemma follows from the fact that the portion of the curve $\Gamma$ located inside $A_{i}^{D}$ has a length of at least $L_{i}$. To see this, notice that each receiver $D_{i}^{(j)}$ is connected to $S_{i}$ via a portion of the $\Gamma$ curve. If part of this portion goes outside of $A_{i}^{D}$, we replace it with a line segment from $D_{i}^{(j)}$ to $S_{i}$ as shown in Fig. 4. Otherwise, no change is made to the portion. This procedure leads to a new curve $\hat{\Gamma}$ which connects $S_{i}$ to all receivers and which is located entirely inside $A_{i}^{D}$. It is straightforward to show that the size of $\hat{\Gamma}$ is not less than the part of $\Gamma$ located inside $A_{i}^{D}$. Also, since $\hat{\Gamma}$ connects $S_{i}$ to all receivers, its size is at least $L_{i}$.

Proof of Theorem 5.5: Now, consider an arbitrary set of multicast transmissions $Q$ at a given time instant $\tau$ (see Section III-B). Denote the EMST curve by $\Gamma_{\text {EMST }}$. From Lemmata 5.2 and 5.6 it follows that

$$
\begin{aligned}
\sum_{i \in Q} L_{i} W_{i} & \leq \sum_{i \in Q} \int_{\Gamma_{\mathrm{EMST}}} \phi_{i}(X) d \ell=\int_{\Gamma_{\mathrm{EMST}}} \sum_{i \in Q} \phi_{i}(X) d \ell \\
& \leq \int_{\Gamma_{\mathrm{EMST}}} M W_{o} d \ell=M W_{o} L_{\mathrm{EMST}}
\end{aligned}
$$

We point out that Theorem 5.5 can be established also for Protocol model 1 and the Generalized Physical Model at the cost of an extra constant factor in the upper bound. For a proof, define the arena to be $A_{i}^{S}=\left\{X:\left|X-S_{i}\right| \leq 2 l_{i}\right\}$. We omit the details for lack of space.

From the above results, we have $C_{T} \leq C_{M T}=O\left(W_{o} \cdot L_{\mathrm{EMST}}\right)$ and since $C_{T}=\Omega\left(W_{o} \cdot L_{\mathrm{EMST}} / \log (n)\right)$, we can conclude that $C_{M T} / C_{T}=O(\log (n))$.

Finally, we compute an upper bound on the multicast capacity using the above upper bound on $C_{M T}$.

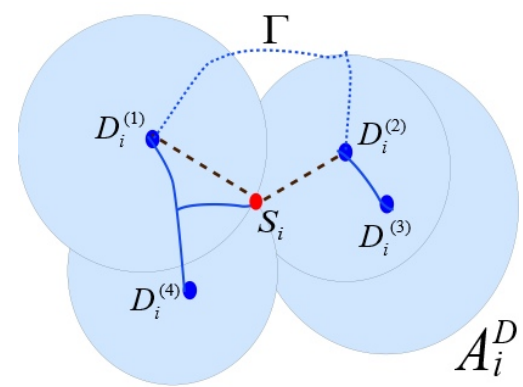

Fig. 4. The EMST curve $\Gamma$ (blue, dashed and solid) and the protection area $A^{D}$ (shaded) of the sender-receiver-tuple $\left(S_{i},\left\{D_{i}^{(1)}, D_{i}^{(2)}, D_{i}^{(3)}, D_{i}^{(4)}\right\}\right)$. A part of $\Gamma$ lies outside $A^{D}$. Therefore, we replace the dashed part of $\Gamma$ by dashed straight lines in black to connect the sender and all receivers by a new curve $\hat{\Gamma}$ (solid blue and dashed black) which is located entirely inside the protection area.

Corollary 5.7: Consider a wireless network with $n$ nodes which are homogeneously distributed in a square area A. Assume multicast sessions from every node to $n_{m}$ randomly selected nodes in the network. Then, the total throughput of the multicast sessions is bounded by

$$
\lambda=O\left(W_{o} M \sqrt{\frac{n}{n_{m}}}\right) \text { a.s. }
$$

as $n \rightarrow \infty$.

Proof of Corollary 5.7: Based on the Theorem 5.5, $C_{T M}=$ $O\left(W_{o} M L_{\mathrm{EMST}}\right)$ and we know that a.s. $L_{\mathrm{EMST}}=\Theta(\sqrt{A n})$. From the definition of multicast transport capacity, we have $\lambda L_{\mathrm{EST}} \leq C_{T M}$, where $L_{\mathrm{EST}}$ is the average length of the EST of the multicast sessions. It has been shown that $L_{\mathrm{EST}}=$ $\Omega\left(\sqrt{A n_{m}}\right)$ a.s. [41]. From the above inequalities we conclude that $\lambda=O\left(W_{o} M \sqrt{A n} / \sqrt{A n_{m}}\right)$

We point out that similar upper bounds on multicast capacity based on the length of the Euclidean Steiner Tree have been proposed in other work [6], [11], [12]. However, no rigorous mathematical proof has been given so far. Our novel analytical proof uses an upper bound on the multicast transport capacity to bound the multicast throughput capacity of the network. In the next section, we present yet another rigorous methodology based on arenas which allows to find an upper bound on the multicast capacity.

\section{Bounds ON MULTiCAST CAPACITY}

So far, we dealt with transport capacity. In this section, we generalize the concept of transmission arena-rate functions and establish novel capacity bounds for multicast flows.

\section{A. Diffusion-span}

In order to tackle the multicast capacity we define a quantity analogous to the curve integral (20). While the curve integral is tailored to the one-dimensional transport of information inherent to unicast flows, this new quantity should be tailored to the spatial distribution of information inherent to multicast flows. Thus motivated we define diffusion-span of a given particular multicast bit $b$ by considering the space integral

$$
\sigma_{b}^{\Omega}=\sum_{i \in \mathcal{H}_{b}} \int_{\Omega} \mathbb{I}_{A_{i}}(X) d X
$$


where $\mathcal{H}_{b}$ is the set of transmissions (whether one-to-one or one-to-many) which transport the bit $b$ of the multicast flow and $\Omega \subset \mathbb{R}^{d}$ is an arbitrary Borel set.

We may think of the diffusion-span $\sigma_{b}$ as the volume of space inside $\Omega$ which is "filled" or "spanned" for transporting a particular bit. Clearly, it depends on the locations of the transmissions (routing paths) and the shape of $\Omega$. The shape of $\Omega$ is chosen properly such that Lemma 6.1 and Theorem 6.2 provide upper bounds on the capacity. For many scenarios, setting $\Omega$ to be a large $d=1,2,3$-dimensional sphere (or cube) containing the transmission arenas, the diffusion-span can be easily computed as $\sigma_{b}^{\Omega}=\pi_{d} \sum_{i \in \mathcal{H}_{b}} l_{i}^{d}$ where $\pi_{d}$ is the volume of unit sphere in $\mathbb{R}^{d}$ and $l_{i}$ is the length of $i$ 'th transmission on the paths.

\section{B. Multicast Capacity Bounds}

Note that we sum over successive hops along a path $\mathcal{H}_{b}$ in (26), as opposed to a set of simultaneous transmissions as in (11). Thus, we average over time in order to obtain bounds:

Lemma 6.1: Assume that data bits $\left\{b_{1}, b_{2}, \ldots\right\}$ have been generated and transported to their destinations in time interval $[0, T]$. Then,

$$
\frac{1}{T} \sum_{b_{k}} \sigma_{b_{k}}^{\Omega} \leq M W_{o}|\Omega|
$$

where $|\Omega|=\int_{\Omega} d X$ is the volume of $\Omega$.

Now, we consider a multicast session $\mathfrak{s}$ in the network for which we have at our disposal a lower bound $s_{\mathfrak{\xi}}$ on the long term average $\sigma_{b_{k}}^{\Omega}$; again, $b_{k}$ denote the bits transported by the session. Applying $s_{\mathfrak{5}}$ over the space that is occupied by the transmissions which transport the information bits from the source to the destinations we are able to establish the following result:

Theorem 6.2: Assume that $s_{\mathfrak{s}} \leq\left(\sum_{b_{k}} \sigma_{b_{k}}^{\Omega}\right) /\left(\sum_{b_{k}} 1\right)$ as $T \rightarrow$ $\infty$ where $\left\{b_{k}\right\}_{k}$ are the transported bits under session $\mathfrak{s}$. Let $\lambda_{\mathfrak{5}}$ be the rate of successfully transported bits of the session. Then

$$
\lambda_{\mathfrak{s}} \leq M W_{o} \frac{|\Omega|}{s_{\mathfrak{s}}}
$$

Proof of Theorem 6.2: Consider the transported bits in the time interval $\left[0, T-T_{D}\right]\left(T>>T_{D}\right)$ where $T_{D}$ is the maximum delay for transporting a bit under session $\mathfrak{s}$. From the assumption, the number of the successfully transported bits in this interval is $\lambda_{\mathfrak{s}}\left(T-T_{D}\right)$. Then, we have

$$
\begin{aligned}
\left(T-T_{D}\right) \lambda_{\mathfrak{s}} \cdot s_{\mathfrak{5}} & =\sum_{k=1}^{\lambda_{\mathfrak{s}}\left(T-T_{D}\right)} s_{\mathfrak{s}} \leq \sum_{k=1}^{\lambda_{\mathfrak{s}}\left(T-T_{D}\right)} \sigma_{b_{k}}^{\Omega} \\
& =\sum_{k=1}^{\lambda_{\mathfrak{s}}\left(T-T_{D}\right)} \sum_{i \in \mathcal{H}_{b_{k}}} \int_{\Omega} \mathbb{I}_{A_{i}}(X) d X \\
& \leq T \cdot \max _{Q} \sum_{j \in Q} \int_{\Omega} \phi_{j}(X) d X \\
& =T \cdot \int_{\Omega}\left(\max _{Q} \sum_{j \in Q} \phi_{j}(X)\right) d X
\end{aligned}
$$

$$
\leq T M W_{o} \cdot \int_{\Omega} d X
$$

Letting $T \rightarrow \infty$ completes the proof.

As an example, consider a wireless network with grid topology and random multicast flows. Then, the multicast capacity can be bound using Theorem 6.2 as stated in the next corollary.

Corollary 6.3: Consider a wireless network with $n$ nodes which are distributed as a grid in a square area A. Assume a multicast session 5 from a source node to $n_{m}$ randomly selected nodes in the network. Then, as $n \rightarrow \infty$,

$$
\lambda_{\mathfrak{s}}=O\left(W_{o} M \sqrt{\frac{n}{n_{m}}}\right) \text { a.s. }
$$

as $n \rightarrow \infty$.

Notably, our novel framework can provide further results on multicast capacity. This framework is used to in our previous work [9], to derive a new bound on the multicast capacity of large homogeneous wireless networks.

\section{Cutset Bounds BASED ON TOPOLOGY}

Calculating cutset bounds for wireless networks usually leads to a complicated maximization problem in terms of channel parameters among various nodes [43], [44]. In this section, we present a method for computing novel cutset bounds and capacity bounds using our arena framework. While the method developed here lends itself to tackle much more general problems, we address two particular cases for illustration purposes: (i) bounding the capacity region of the rates of simultaneous fixed-path flows (ii) bounding the maximum aggregate data rate carried between two parts of a wireless network. Due to lack of space, we constrain this section to the cutset bound. Further results can be found in our earlier work [31].

\section{A. Bounding Average Rates via Arenas}

Let $\mathcal{N}$ denote the set of nodes which are used as senders at certain times along the routes of the a number of flows. For each such sender $S_{i} \in \mathcal{N}$, let $\mathcal{T}_{i}$ denote the set of time instants during which it is successfully sending. Finally, let $M$ denote the arena-bound under the channel model put in place. Then, the defining inequality of (11) implies for any fixed time instant $\tau$ :

$$
\sum_{S_{i} \in \mathcal{N}} W_{i} \mathbb{I}_{A_{i}}(X) \cdot \mathbb{I}_{\mathcal{T}_{i}}(\tau) \leq M \cdot W_{o}
$$

Fixing the location $X$ and averaging over the time interval $[0, T]$ we find

$$
\sum_{S_{i} \in \mathcal{N}}<W_{i}>\mathbb{I}_{A_{i}}(X) \leq M \cdot W_{o},
$$

where $\left\langle W_{i}\right\rangle:=\frac{1}{T} \int_{0}^{T} W_{i} \cdot \mathbb{I}_{\mathcal{T}_{i}}(\tau) d \tau$ is the average rate at which the sender $S_{i}$ transmits data.

In this section we exploit this bound by considering locations $X$ where we expect the most restrictive conditions in the network, i.e., where a large number of arenas overlap, in order to get effective upper bounds on the average rates. 


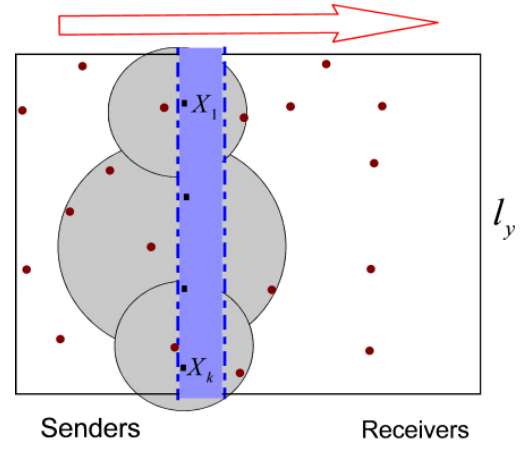

Fig. 5. Computing a cutset bound on the flow from left to right in the rectangular area $l_{x} * l_{y}$.

\section{B. Maximum Flow Rate across a Rectangular Region}

Here, we study the maximum data flow rate between two parts in a network in a special case, namely for the flow rate of one side of a rectangular area to the other as shown in Fig. 5. We assume that there is no single node in the rectangular area able to relay information directly from nodes on the left side to nodes on the right side. Denoting the length and width of the rectangle by $l_{\mathrm{x}}$ and $l_{\mathrm{y}}$ we find that the maximum flow rate from left to right is bounded by $M W_{o} \cdot\left\lceil l_{y} / 2 l_{x}\right\rceil$.

For the proof, consider points $X_{1}, X_{2}, \ldots, X_{k}$ close to the left side of the rectangle area in distance $2 l_{x}$ from each other. The number of such points is $k=\left\lceil l_{y} / 2 l_{x}\right\rceil$. Any transmission from the left to the right side of the rectangle contains at least one of these points $X_{j}$ in its arena $\left(A_{i}^{S}\right)$. Summing (31) over all $X_{k}$, the sum of transmission rates becomes bounded by $k \cdot M W_{o}$.

In general, this framework can be used to bound the maximum output/input flow rate of a set of nodes $\mathcal{U}$ in a wireless network. First, we find a minimal set of points $\left\{X_{1}, \ldots, X_{k}\right\}$ in the space such that every transmission between $\mathcal{U}$ and the rest of the nodes include at least one of selected points in its arena. Then, by applying (31) on each point, we show that the maximum flow rate by $k M W_{o}$. Obviously, smaller $k$ provide tighter bounds on the maximum flow rate.

Note that in the special case where $l_{\mathrm{y}}=l_{\mathrm{x}}$ our results covers the earlier [45]. In [9], the above method is used to bound the maximum flow rate toward a set of relatively isolated nodes in a large homogeneous network. Based on this, a new upper bound is derived for multicast capacity.

\section{Maximum Flow Rate across Border of Different Densities}

Sometimes it is useful to introduce a virtual gap in a network in form of a rectangular region in order to find a cutset bound. Fig. 6 depicts an interesting scenario where this approach proves useful. Here, the density of nodes decreases from $\rho_{1}$ to $\rho_{2}$ along a border line. We define a virtual gap in form of a rectangle with $l_{\mathrm{y}}$ equal to the length of the border line, with $x_{x}=1 / \sqrt{2 \rho_{2}}$ and position it inside the area with lower density $\rho_{2}$ just adjacent to the border line. Then the number of nodes inside the virtual gap is approximately $\rho_{2} l_{\mathrm{x}} l_{\mathrm{y}}=l_{\mathrm{y}} \sqrt{\rho_{2} / 2}$. Therefore, the maximum flow rate which enters the virtual gap is bounded by $M W_{o} l_{y} \sqrt{\rho_{2} / 2}$. Also, from the last result,

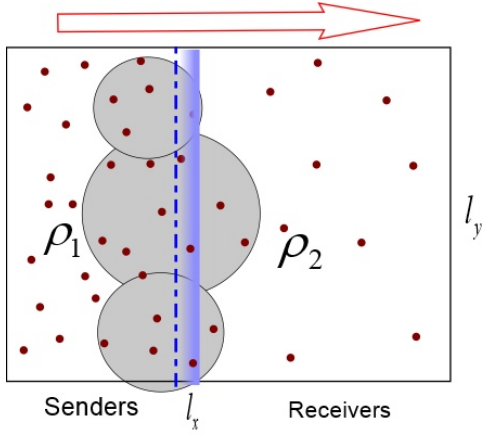

Fig. 6. Computing a cutset bound on the flow that goes from high-density $\left(\rho_{1}\right)$ area to low-density area $\left(\rho_{2}\right)$.

the maximum flow rate that passes through the virtual gap is bounded by $M W_{o} l_{y} /\left(2 l_{x}\right)=M W_{o} l_{y} \sqrt{\rho_{2} / 2}$. Therefore, the total maximum flow rate passing across the border line is bounded by $M W_{o} l_{y} \sqrt{2 \rho_{2}}$.

\section{CONCLUSION AND FUtURE WORK}

We introduced the novel concept of transmission arenas which allows one to study the effect of topology and traffic patterns on the capacity of wireless networks in much more detail than existing work. Note that the networks are not assumed to be homogeneous. The key property behind all results is the existence of an arena-bound which imposes limitations on simultaneous transmissions in a compact, analytically tractable way. The simplicity and effectiveness of our methodology comes from the fact that we take a spatial approach where arena-rate functions indicate the impact at every location in the network space caused by simultaneous transmissions.

The arena-bound imposed at every location and time is used in three ways: 1) fixing a location and averaging over time we find cutset bounds on simultaneous flows which are more accurate or computationally less demanding than standard methods. 2) fixing time and averaging along appropriate curves we provide novel bounds on the transport capacity in terms of the network's Euclidean Minimum Spanning Tree or Euclidean Steiner Tree. They are more accurate than the existing bounds since they do not rest on the assumption of a homogeneous network. 3) fixing time and averaging over space we introduce novel bounds on multicast sessions. Our work applies to the three classical channel models, the Protocol, the Physical and the Generalized Physical Models in all dimensions of space. Our approach also motivates future studies which aim to investigate network capacity using alternative arena-rate functions and allows for investigating wireless networks with multi-channel, directional antennas and hybrid backbones.

\section{APPENDIX}

Proof of Lemma 4.1: Consider an arbitrary point $X \in \mathbb{R}^{d}$ at time $\tau$ and assume that $\phi_{1}(X), \ldots, \phi_{m}(X)>0$. If $m=1$ the upper bound is trivial. So, assume that $m>1$ and $l_{1} \leq l_{2} \leq$ $\ldots \leq l_{m}$ without lack of generality (see Fig. 7). We find the arena-bound $M$ for the models separately as follows: 
Protocol Model (1): First we obtain an inequality through double application of triangular inequality. Consider two transmitters $S_{j}$ and $S_{k}$, then

$$
\begin{aligned}
\left|S_{j}-S_{k}\right| & \geq \frac{1}{2}\left(\left|S_{j}-D_{k}\right|-\left|S_{k}-D_{k}\right|+\left|S_{k}-D_{j}\right|-\left|S_{j}-D_{j}\right|\right) \\
& \geq \frac{1}{2}\left((1+\Delta)\left(l_{j}+l_{k}\right)-l_{j}-l_{k}\right)=\frac{\Delta}{2}\left(l_{j}+l_{k}\right)
\end{aligned}
$$

The inequality shows that the balls with radiuses $\Delta l_{j} / 2$ around the transmitters $S_{j}$ are disjoint in $\mathbb{R}^{d}$ for $j=\{1,2, \ldots, m\}$. Now, we consider a bigger ball with radius $(1+\Delta / 2) l_{m}$ around point $X$, it covers the balls around $S_{1}, \ldots, S_{m}$. So, we have

$$
\sum_{j=1}^{m} \pi_{d}\left(\Delta l_{j} / 2\right)^{d}<\pi_{d}(1+\Delta / 2)^{d} l_{m}^{d}
$$

where $\pi_{d}$ is the volume of unit sphere in $\mathbb{R}^{d}$.

On the other hand, we assumed that $D_{1}$ receives successfully, (i.e. $\phi_{1}(X)>0$ ). From the channel model we have

$$
\begin{aligned}
(1+\Delta) l_{m} & <\left|S_{m}-D_{1}\right| \\
& \leq\left|S_{m}-X\right|+\left|X-S_{1}\right|+\left|S_{1}-D_{1}\right| \\
& \leq l_{m}+2 l_{1}
\end{aligned}
$$

From above it follows that

$$
l_{m} \leq \frac{2}{\Delta} l_{1} .
$$

Clearly, if $\Delta>2$ then (33) is a contradiction which shows that $m=1$ and we set $M=1$ for this case. If $\Delta \leq 2$, (32) and (33) imply that $m\left(\Delta l_{1} / 2\right)^{d}<\left(\frac{2(1+\Delta / 2)}{\Delta} l_{1}\right)^{d}$, hence $m<\frac{(4+2 \Delta)^{d}}{\Delta^{2 d}}$. Therefore, we set $M=\left\lceil\frac{(4+2 \Delta)^{d}}{\Delta^{2 d}}-1\right\rceil$.

Protocol Model (2): Here, the problem formulation becomes the same as for Model (1), using $A_{i}^{D}$ instead of $A_{i}^{S}$. So, we obtain the same $M$.

Protocol Model (3): We prove a stronger result here. Consider $\psi_{i}(X)=W_{i} \cdot \mathbb{I}_{\left|X-S_{i}\right| \leq r}$. Clearly, $\phi_{i}(X) \leq \psi_{i}(X)$ when $\phi_{i}(X)$ is defined in terms of arena $A_{i}^{S}$. Now, we show that $\sum_{i} \psi_{i}(X) \leq M \cdot W_{o}$. Similar to the proof of Model 1, we show if $\Delta>2$ then $M=1$. If $\Delta \leq 2$, we consider the balls with radius $\Delta r / 2$ around the transmitters $S_{j}$. These balls are disjoint and contained in the bigger ball with radius $(1+\Delta / 2) r$ around point $X$. It follows that $m<\pi_{d}(1+\Delta / 2)^{d} r^{d} / \pi_{d}(\Delta r / 2)^{d}=\frac{(2+\Delta)^{d}}{\Delta^{d}}$. Therefore, we can set $M=\left\lceil\frac{(2+\Delta)^{d}}{\Delta^{d}}-1\right\rceil$.

Note that the same $M$ is obtained if we use this method for arena $A_{i}^{D}$.

Physical Model: Here, we bound the SINR of receiver $D_{m}$ as the following

$$
\left|S_{k}-D_{m}\right| \leq\left|S_{k}-X\right|+\left|X-S_{m}\right|+\left|S_{m}-D_{m}\right| \leq 3 l_{m}
$$
So,

$$
\begin{aligned}
\operatorname{SINR}_{m} & <\frac{P_{m} l_{m}^{-\alpha}}{\sum_{k=1}^{m-1} P_{k}\left|S_{k}-D_{m}\right|^{-\alpha}} \leq \frac{P_{\max }}{P_{\min }} \frac{l_{m}^{-\alpha}}{\sum_{k=1}^{m-1}\left|S_{k}-D_{m}\right|^{-\alpha}} \\
& \leq \frac{P_{\max }}{P_{\min }} \frac{l_{m}^{-\alpha}}{\sum_{k=1}^{m-1}\left(3 l_{m}\right)^{-\alpha}}=\frac{3^{\alpha} P_{\max }}{(m-1) P_{\min }}
\end{aligned}
$$

Since, $\operatorname{SINR}_{m} \geq \beta$, it follows that $\beta<\frac{3^{\alpha} P_{\max }}{(m-1) P_{\min }}$, hence $m<$ $\frac{3^{\alpha} P_{\max }}{\beta P_{\min }}+1$. Therefore, we can set $M=\left\lceil\frac{3^{\alpha} P_{\max }}{\beta P_{\min }}\right\rceil$.

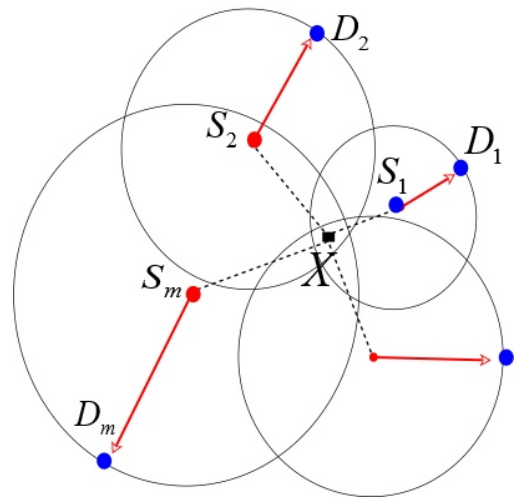

Fig. 7. Point $X$ is in the intersection of the disturbance areas of some simultaneous transmission.

Proof of Lemma 4.2: Similar to the proof of Lemma 4.1, consider $l_{1} \leq l_{2} \leq \ldots \leq l_{m}$. If $m=1$ the upper bound is trivial, so let us consider the case $m>1$. We bound the SINR of receiver $D_{j}$ for a $1<j \leq m$ in the following way

$$
\left|S_{k}-D_{j}\right| \leq\left|S_{k}-X\right|+\left|X-S_{j}\right|+\left|S_{j}-D_{j}\right| \leq 3 l_{j}
$$

for all $k<j$. It follows that

$$
\begin{aligned}
\mathrm{SINR}_{j} & <\frac{P_{j} l_{j}^{-\alpha}}{\sum_{k=1}^{j-1} P_{k}\left|S_{k}-D_{j}\right|^{-\alpha}} \\
& \leq \frac{P_{\max }}{P_{\min }} \frac{l_{j}^{-\alpha}}{\sum_{k=1}^{j-1}\left|S_{k}-D_{j}\right|^{-\alpha}} \\
& \leq \frac{P_{\max }}{P_{\min }} \frac{l_{j}^{-\alpha}}{\sum_{k=1}^{j-1}\left(3 l_{j}\right)^{-\alpha}}=\frac{3^{\alpha} P_{\max }}{(j-1) P_{\min }}
\end{aligned}
$$

So,

$$
W_{j} \leq B \log _{2}\left(1+\operatorname{SINR}_{j}\right) \leq B \frac{3^{\alpha} P_{\text {max }}}{(j-1) P_{\text {min }}} \log _{2}(e)
$$

Therefore, ${ }^{3}$

$$
\begin{aligned}
\sum \phi_{i}(X) & \leq W_{1}+\sum_{j=2}^{m} B \frac{3^{\alpha} P_{\max }}{(j-1) P_{\min }} \log _{2}(e) \\
& \leq \max _{i}\left(W_{i}\right)+B \frac{3^{\alpha} P_{\max }}{P_{\min }} \log _{2}(e) \sum_{j=1}^{\# Q-1} \frac{1}{j} \\
& \leq \max _{i}\left(W_{i}\right)+B \cdot \frac{3^{\alpha} P_{\max } \log _{2}(e \cdot \# Q)}{P_{\min }}
\end{aligned}
$$

For the second upper bound, we bound $\operatorname{SINR}_{j}$ for all $j$

$$
\left|S_{k}-D_{j}\right| \leq\left|S_{k}-X\right|+\left|X-S_{j}\right|+\left|S_{j}-D_{j}\right| \leq 3 l_{\max }
$$

So,

$$
\begin{aligned}
\mathrm{SINR}_{j} & <\frac{P_{j} l_{j}^{-\alpha}}{\sum_{k=1, k \neq j}^{m} P_{k}\left|S_{k}-D_{j}\right|^{-\alpha}} \leq \frac{P_{\max }}{P_{\min }} \frac{l_{j}^{-\alpha}}{\sum_{k=1, k \neq j}^{m}\left|S_{k}-D_{j}\right|^{-\alpha}} \\
& \leq \frac{P_{\max }}{P_{\min }} \frac{l_{\min }{ }^{-\alpha}}{\sum_{k=1, k \neq j}^{m}\left(3 l_{\max }\right)^{-\alpha}}=\frac{P_{\max }}{(m-1) P_{\min }}\left(\frac{3 l_{\max }}{l_{\min }}\right)^{\alpha}
\end{aligned}
$$

${ }^{3}$ We use the following inequality $\sum_{j=1}^{m} \frac{1}{j} \leq 1+\log (m)$ 
Then

$$
\begin{aligned}
\sum \phi_{i}(X) & \leq \sum_{j=1}^{m} B \log _{2}\left(1+\operatorname{SINR}_{j}\right) \\
& \leq B m \log _{2}\left(1+\frac{P_{\max }}{(m-1) P_{\min }}\left(\frac{3 l_{\max }}{l_{\min }}\right)^{\alpha}\right) \\
& \leq 2 \log _{2}(e) B \frac{P_{\max }}{P_{\min }}\left(\frac{3 l_{\max }}{l_{\min }}\right)^{\alpha}
\end{aligned}
$$

Note that the same $M$ is obtained for $A_{i}^{D}$ using this method. Proof of Corollary 5.3: From Theorem 5.1, it is enough to show that $L_{\mathrm{EMST}} \leq K_{d} \sqrt[d]{V n^{d-1}}$. In one dimensional space, the inequality in obvious, because $L_{\mathrm{EMST}}$ is the diameter of the network. In two and three dimensional space we use induction for all $n>1$. We set $K_{d}=3 d$, and prove the inequality for $n=2,3$. Obviously, the maximum distance between two nodes is less than the diameter of the cube $(\sqrt{d} \sqrt[d]{V})$. So, $L_{\text {EMST }} \leq$ $(n-1) \sqrt{d} \sqrt[d]{V}<3 d \sqrt[d]{V n^{d-1}}$ (only for $n=2,3$ ).

For $n>1$, we assume the inequality for $k \leq n-1$ and we prove for $k=n$. Consider the balls with radius $1.5 \sqrt[d]{\frac{V}{n}}$ around all nodes. At least $\pi_{d}\left(1.5 \sqrt[d]{\frac{V}{n}}\right)^{d} / 2^{d}$ volume of each ball is located inside $V$. The sum of the volumes of these balls inside $V$ is larger than $n \cdot \pi_{d}\left(1.5 \sqrt[d]{\frac{V}{n}}\right)^{d} / 2^{d}=\frac{\pi_{d} 3^{d}}{4^{d}} V>V$. Therefore, at least two of these balls are not disjoint that means there exists a pair of nodes within distance $2 * 1.5 \sqrt[d]{\frac{V}{n}}=3 \sqrt[d]{\frac{V}{n}}$. We eliminate one of these nodes from the set of the nodes. For the remaining $n-1$, we know the length of the EMST is less than $3 d \sqrt[d]{V(n-1)^{d-1}}$. By adding the eliminated node and connecting it to its closet neighbor we build an spanning tree with length of less than $3 d \sqrt[d]{V(n-1)^{d-1}}+3 \sqrt[d]{\frac{V}{n}}<3 d \sqrt[d]{V n^{d-1}}$ (to prove this inequality, show that $d(1-1 / n)^{\frac{d-1}{d}}+1 / n<d$ using $0<1 / n<1)$. Therefore, $L_{\text {EMST }} \leq 3 d \sqrt[d]{V n^{d-1}}$.

Proof of Theorem 5.4: We construct a time scheduling and traffic pattern along some edges of the EMST which achieves the lower bound. We consider $d=1$ and $d \geq 2$ cases separately.

When $d=1$, the EMST is the line segment between the two most remote nodes. Due to the well-connectivity assumption, data can be transported between the farthest nodes at rate $\epsilon W_{o}$. This gives us a traffic pattern and time scheduling with a transport capacity larger than $K_{1} W_{o} L_{\text {EMST }}$ where $K_{1}=\epsilon$. When $d \geq 2$, we proceed in five steps.

Step 1: We select the edges of the EMST with the length of at least $L_{\mathrm{EMST}} / 2 n$. Denote the set of selected edges by $l_{1} \leq \ldots \leq l_{m}$. We have the following inequality for $\sum l_{i}$ :

$$
\sum_{i=1}^{m} l_{i} \geq L_{\mathrm{EMST}}-(n-m) L_{\mathrm{EMST}} / 2 n>L_{\mathrm{EMST}} / 2
$$

Step 2: We partition $\left\{l_{1}, \ldots, l_{m}\right\}$ into $u$ sets $C_{1}, \ldots, C_{u}$ where $C_{j}=\left\{l_{i}: 2^{-j} L_{\mathrm{EMST}}<l_{i} \leq 2^{-j+1} L_{\mathrm{EMST}}\right\}$. Note that $u=$ $\left\lceil\log _{2}(2 n)\right\rceil$. We divide the time into $u$ equal time slots. During the $j^{\text {th }}$ time slot, the data is transmitted only along the edges of $C_{j}$. Next, we do steps 3 to 5 at time slots of $C_{j}$ for all $j=1,2, \ldots, u$.
Step 3: We divide the space into cube cells with side size $r_{j}=2^{-j+1} L_{\text {EMST }}$ such that the coordinates of their centers are $\left(i_{1} r_{j}, i_{2} r_{j}, \ldots, i_{d} r_{j}\right)$ for $i_{1}, \ldots, i_{d} \in \mathbb{Z}$ (see Fig. 8).

Such a cellular structure has two properties. (i) If a vertex of an edge $l_{i} \in C_{j}$ is located in a cell, the other vertex of $l_{i}$ in located either in the same cell or in one of the $3^{d}-1$ neighbor cells around it. (ii) The number of edges in set $C_{j}$ with at least one of their vertices located in the same cell is bounded by a constant.

For a proof, assume that there are $k_{1}$ edges of $C_{j}$ such that at least one on their vertices is inside the cell. Since the lengths of the edges are less than $r_{j}$, the edges are located inside the cube with side size $3 r_{j}$ formed by the cell and the areas its neighbor cells. By Corollary 5.3, the length of the EMST of the vertices of these edges is less than $K_{d} \sqrt[d]{\left(2 k_{1}\right)^{d-1} 3^{d} r_{j}^{d}}$. This is an upper bound for the sum of the lengths of the edges, because if we can connect all these vertices with a spanning tree with smaller length, then we can reduce the length of the EMST of the network which is a contradiction. On the other hand, the length of the edges of $C_{j}$ is at least $r_{j} / 2$, so the sum of the length of the edges is larger than $k_{1} r_{j} / 2$. We conclude that $k_{1} r_{j} / 2<3 K_{d} r_{j} \sqrt[d]{\left(2 k_{1}\right)^{d-1}}$, and $k_{1}<2^{2 d-1}\left(3 K_{d}\right)^{d}$.

Step 4: We assign color $C\left(\rho_{q_{1}}, \rho_{q_{2}}, \ldots, \rho_{q_{d}}\right)$ to the cell with center coordinates of $\left(q_{1} r_{j}, q_{2} r_{j}, \ldots, q_{d} r_{j}\right)$, where $\rho_{q}=$ $q(\mathrm{mod}) k_{2}$. For time scheduling, we divide the time slot of $C_{j}$ into $k_{2}^{d}$ subslots and we assign one color to each subslot. At every time subslot only the cells with the corresponding color can be active. A cell is called active when a vertex inside the cell transmits.

The constant $k_{2}$ is chosen large enough such that data can be transmitted along some edges $C_{j}$ in different cells simultaneously and with rate $W_{o}$. Using Lemma 9.1, we can set $k_{2}$ for different channel models as (35).

Lemma 9.1: The value of $k_{2}$ for different channel models can be chosen as

$$
k_{2}= \begin{cases}\lceil 3+\Delta\rceil & \text { Protoc. Models 1, 2, } \\ \left\lceil 2+\left(\frac{\beta}{1-\varepsilon} \sum_{Q \in \mathbb{Z}_{\circ}^{d}}|Q|^{-\alpha}\right)^{1 / \alpha}\right\rceil & \text { Phys. Model, } \\ \left\lceil 2+\left(\frac{2^{\epsilon}-1}{1-\varepsilon} \sum_{Q \in \mathbb{Z}_{\circ}^{d}}|Q|^{-\alpha}\right)^{1 / \alpha}\right\rceil & \text { Gener. Phys. Model. }\end{cases}
$$

where $\mathbb{Z}_{\circ}^{d}:=\mathbb{Z}^{d} \backslash\{(0, \ldots, 0)\}$.

Proof of Lemma 9.1: We prove the lemma for Protocol Model 1 and 2 and Physical models separately.

From the definition of $C_{j}$, the distance between each sender and receiver pair is less than $r_{j}$. Therefore, the circular interference area created by a transmission has radius of at most $(1+\Delta) r_{j}$. If we set the distance (of centers) of two cells which contain to simultaneous sender larger than $(3+\Delta) r_{j}$, then the distance between each sender from the receiver of the other sender is larger than $(1+\Delta) r_{j}$. Therefore, when $k_{2} \geq 3+\Delta$, then all simultaneous transmissions are successful under Protocol Model 1 and 2 .

Next, we consider the Physical and the Generalized Physical Model. Note that the well-connectivity assumption restricts the length of the edges of the EMST and guarantees that $k_{2}$ is finite. For a proof, consider two vertices (nodes) of the edge $l_{m}$ (the edge maximum size). Any path which connects these two nodes has an edge with size larger than or equal 
to $l_{m}$, otherwise we can reduce the length of the EMST. So, transmissions along distance $l_{m}$ with maximum power $\varepsilon P_{\max }$ and rate $\epsilon W_{o}$ must be feasible. It follows that $l_{i} \leq l_{m} \leq$ $\left(\frac{\varepsilon P_{\max }}{\beta N}\right)^{1 / \alpha}$ in Physical Model, and $l_{i} \leq l_{m} \leq\left(\frac{\varepsilon P_{\max }}{\left(2^{\epsilon}-1\right) N_{0} B}\right)^{1 / \alpha}$ in Generalized Physical Model. Later, we apply these inequalities for computing parameter $k_{2}$ in the cellular structure.

Recall that the simultaneous transmitters are in the different cells with the same color. For example, consider a transmitter $S_{i}$ in cell $(0, \ldots, 0)$ with a receiver $D_{i}$ within distance $r_{j}$. Any simultaneous transmitter $S_{i^{\prime}}$ must lie in a cell $\left(q_{1} k_{2}, q_{2} k_{2}, \ldots, q_{d} k_{2}\right)$ where $Q=\left(q_{1}, \ldots, q_{d}\right) \in \mathbb{Z}_{\circ}^{d}$. The distance between the sender $S_{i^{\prime}}$ and the receiver $D_{i}$ is then at least $\sqrt{q_{1}^{2}+\ldots+q_{d}^{2}} k_{2} r_{j}-2 r_{j}$, and since $|Q| \geq 1$, at least $\geq r_{j}\left(k_{2}-2\right)|Q|$. Thus,

$$
\begin{aligned}
\operatorname{SINR} & \geq \frac{P_{\max } l_{i}^{-\alpha}}{N+\sum_{Q \in \mathbb{Z}_{o}^{d}} P_{\max }\left(r_{j} k_{2}|Q|-2 r_{j}\right)^{-\alpha}} \\
& \geq \frac{P_{\max } l_{i}^{-\alpha}}{N+\sum_{Q \in \mathbb{Z}_{o}^{d}} P_{\max }\left(r_{j}\left(k_{2}-2\right)|Q|^{-\alpha}\right.} \\
& \geq \frac{1}{\frac{N}{P_{\max }} l_{i}^{\alpha}+\left(k_{2}-2\right)^{-\alpha} \sum_{Q \in \mathbb{Z}_{o}^{d}}|Q|^{-\alpha}} \\
& \geq \frac{1}{\frac{N}{P_{\max }} l_{m}^{\alpha}+\left(k_{2}-2\right)^{-\alpha} \sum_{Q \in \mathbb{Z}_{o}^{d}}|Q|^{-\alpha}} \\
& \frac{1}{\varepsilon / \beta+\left(k_{2}-2\right)^{-\alpha} \sum_{Q \in \mathbb{Z}_{o}^{d}}|Q|^{-\alpha}}
\end{aligned}
$$

Next, we set $k_{2}$ large enough such the computed lower bound of SINR is larger than $\beta$. Then we have

$$
k_{2} \geq 2+\left(\frac{\beta}{1-\varepsilon} \sum_{Q \in \mathbb{Z}_{o}^{d}}|Q|^{-\alpha}\right)^{1 / \alpha}
$$

We apply the same techniques for Generalized Physical Model. Then, we can show that the following $k_{2}$ satisfies the lemma.

$$
k_{2} \geq 2+\left(\frac{2^{\epsilon}-1}{1-\varepsilon} \sum_{Q \in \mathbb{Z}_{\circ}^{d}}|Q|^{-\alpha}\right)^{1 / \alpha}
$$

Note that we use the assumption $\alpha>d$ to show that $\sum_{Q \in \mathbb{Z}_{o}^{d}}|Q|^{-\alpha}$ converges and $k_{2}$ is a finite number.

Step 5: Since, we need at most $k_{1}$ transmission along the edges of $C_{j}$ for each cell, we divide the subslot of the cell into $k_{1}$ equal subsubslots. In each time subsubslot, data is transmitted along one of the edges.

For this traffic pattern and time scheduling scheme the average transmission rate along the edges $\left\{l_{1}, \ldots, l_{m}\right\}$ equal to $W_{o} /\left(k_{1} k_{2}^{d} u\right)$. This yields a transport capacity of at least:

$$
\begin{aligned}
C_{T} & \geq W_{o} /\left(k_{1} k_{2}^{d} u\right) \sum_{i=1}^{m} l_{i} \\
& >W_{o} /\left(k_{1} k_{2}^{d} u\right) \cdot L_{\mathrm{EMST}} / 2 \geq K_{1} W_{o} \cdot L_{\mathrm{EMST}} / \log (n)
\end{aligned}
$$

where $K_{1}$ is a constant number.

Proof of Corollary 6.3: We set $\Omega=A$. Then, we only need to show that setting $s_{\mathfrak{s}}=c A \sqrt{\frac{n_{m}}{n}}$ satisfies the condition of Theorem 6.2, almost surely, as $n$ grows, where $c$ is a constant.

To prove $s_{\mathfrak{5}} \leq\left(\sum_{b_{k}} \sigma_{b_{k}}^{\Omega}\right) /\left(\sum_{b_{k}} 1\right)$ a.s., we show that $s_{\mathfrak{5}} \leq$ $\sigma_{b_{k}}^{\Omega}$ a.s. as $n$ grows. Consider $n_{m}$ random destinations of a

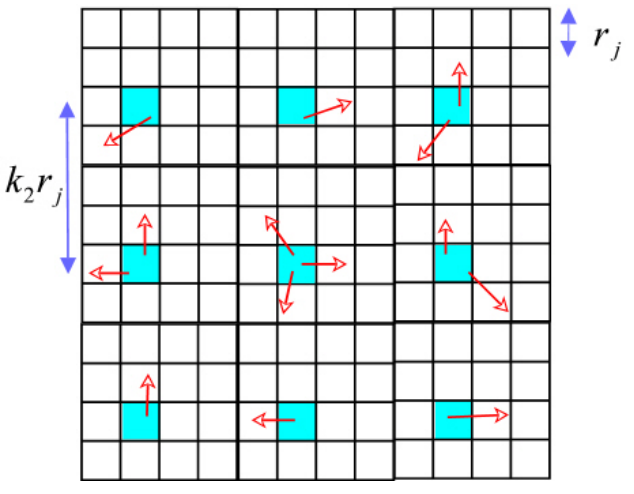

Fig. 8. $k_{2}$ is large enough such that the nodes in different cells with same color can transmit simultaneously with rate $W_{o}$.

particular data bit $b_{k}$. Using probability theory techniques, we can show that the size of maximum independent set has a lower bounds proportional to $n_{m}$, i.e., there exists a constant $c_{1}$ such that \#MIS $\left(2 \sqrt{\frac{A}{n_{m}}}\right)>c_{1} n_{m}$, almost surely, as $n_{m}$ grows (see Lemma 2 in [33]). We draw circles with radius $\sqrt{\frac{A}{n_{m}}}$ around the nodes of MIS. Clearly, the circles are disjoint in the plane. Next, for every transmission, we color all the grid squares (side size $=\sqrt{\frac{A}{n}}$ ) which at least $\frac{\pi}{4}$ of their area is located inside the disturbance area of the transmission to black. It is easy to show that if we connect the neighbor black squares, then a path from the transmitter to the receiver (receivers in one-tomany transmissions) is created. This shows that there exists a path of black squares which connects MIS to a node outside of the circle corresponding to it. So, the number of black squares inside the circle is at least $\left\lfloor\sqrt{\frac{A}{n_{m}}} / \sqrt{\frac{A}{n}}\right\rfloor$. Since the circles are disjoint, we conclude that the number of black squares is larger than $c_{1} n_{m} \cdot\left\lfloor\sqrt{\frac{A}{n_{m}}} / \sqrt{\frac{A}{n}}\right\rfloor \geq c_{2} \sqrt{n n_{m}}$. This shows that the sum of disturbance areas of the transmissions which transport a particular bit is larger than $c_{2} \sqrt{n n_{m}} \frac{\pi}{4} \frac{A}{n}=c A \sqrt{\frac{n_{m}}{n}}$.

\section{REFERENCES}

[1] P. Gupta and P. R. Kumar, "The capacity of wireless networks," IEEE Transactions on Information Theory, vol. 46, no. 2, pp. 388-404, 2000.

[2] M. Grossglauser and D. N. C. Tse, "Mobility increases the capacity of ad-hoc wireless networks," in INFOCOM, 2001.

[3] S. Toumpis, "Capacity bounds for three classes of wireless networks: asymmetric, cluster, and hybrid," in MobiHoc, 2004.

[4] M. Franceschetti, O. Dousse, D. Tse, and P. Thiran, "On the throughput capacity of random wireless networks," IEEE Transactions on Information Theory, vol. 52, no. 6, 2006.

[5] A. Keshavarz-Haddad, V. Ribeiro, and R. Riedi, "Broadcast capacity in multihop wireless networks," in MobiCom, 2006.

[6] X. Y. Li, "Multicast capacity of wireless ad hoc networks," IEEE/ACM Transactions on Networking, vol. 17, no. 3, pp. 950-961, 2009.

[7] S. R. Kulkarni and P. Viswanath, "A deterministic approach to throughput scaling in wireless networks," IEEE Transactions on Information Theory, vol. 50, no. 6, pp. 1041-1049, 2004.

[8] P. R. Jelenković, P. Momčilović, and M. S. Squillante, "Scalability of wireless networks," IEEE/ACM Transactions on Networking, vol. 15, no. 2, pp. 295-308, 2007.

[9] A. Keshavarz-Haddad and R. Riedi, "Multicast capacity of large homogeneous multihop wireless networks," in WiOpt, 2008.

[10] S. Shakkottai, X. Liu, and R. Srikant, "The multicast capacity of large multihop wireless networks," IEEE/ACM Transactions on Networking, vol. 18 , no. 6 , pp. 1691-1700, 2010. 
[11] X. Y. Li, Y. Liu, S. Li, and S. Tang, "Multicast capacity of wireless ad hoc networks under Gaussian channel model," IEEE/ACM Transactions on Networking, vol. 18, no. 4, pp. 1145-1157, 2010.

[12] C. Wang, X. Li, C. Jiang, and S. Tang, "General capacity scaling of wireless networks," in INFOCOM, 2011.

[13] C. Wang, J. Zhou, T. Liu, L. Shao, and H. Yan, "New tight upper bounds on the capacity for general deterministic dissemination in wireless ad hoc networks," in IPCC. IEEE, 2015.

[14] A. Agarwal and P. R. Kumar, "Capacity bounds for ad hoc and hybrid wireless networks," Computer Communication Review, vol. 34, no. 3, pp. $71-81,2004$

[15] P. Gupta and P. R. Kumar, "Internets in the sky: The capacity of three dimensional wireless networks," Communications in Information and Systems, vol. 1, no. 1, pp. 33-50, 2001

[16] P. Kyasanur and N. H. Vaidya, "Capacity of multi-channel wireless networks: impact of number of channels and interfaces," in MobiCom, 2005.

[17] L. Fu and X. Wang, "Multicast scaling law in multi-channel multiradio wireless networks," IEEE Transactions on Parallel and Distributed Systems, vol. PP, 2012.

[18] R. Negi and A. Rajeswaran, "Capacity of ultra wide band wireless ad hoc networks," IEEE Transactions on Wireless Communications, vol. 6 , no. 10, pp. 3816-3824, 2007.

[19] P. Li, C. Zhang, and Y. Fang, "The capacity of wireless ad hoc networks using directional antennas," IEEE Transactions on Mobile Computing, vol. 10, no. 10, pp. 1374-1387, 2011.

[20] X. Chen, W. Huang, X. Wang, and X. Lin, "Multicast capacity in mobile wireless ad hoc network with infrastructure support," in INFOCOM, 2012.

[21] L. Fu, J. Zhang, and X. Wang, "Evolution-cast: Temporal evolution in wireless social networks and its impact on capacity," IEEE Transactions on Parallel and Distributed Systems, vol. 25, no. 10, pp. 2583-2594, 2014.

[22] N. Lu and X. S. Shen, "Scaling laws for throughput capacity and delay in wireless networksâÂTa survey," IEEE Communications Surveys \& Tutorials, vol. 16, no. 2, pp. 642-657, 2014.

[23] S. Toumpis, "Asymptotic capacity bounds for wireless networks with non-uniform traffic," IEEE Transactions on Wireless Communications, vol. 7, no. 6, pp. 2231-2242, 2008.

[24] G. Alfano, M. Garetto, and E. Leonardi, "Capacity scaling of wireless networks with inhomogeneous node density: Upper bounds," IEEE Journal on Selected Areas in Communication, vol. 27, no. 7, pp. 1147-1157, 2009.

[25] V. Martina, M. Garetto, and E. Leonardi, "Capacity scaling of large wireless networks with heterogeneous clusters," Elsevier Science Publishers B. V., vol. 67, no. 11, pp. 1203-1218, 2010.

[26] Q. Peng, X. Wang, and H. Tang, "Heterogeneity increases multicast capacity in clustered network," in INFOCOM, 2011.

[27] C. Wang, C. Jiang, X.-Y. Li, S. Tang, Y. He, X. Mao, and Y. Liu, "Scaling laws of multicast capacity for power-constrained wireless networks under Gaussian channel model," IEEE Transactions on Computers, vol. 61, no. 5, pp. 713-725, 2012.

[28] P. Li and Y. Fang, "On the throughput capacity of heterogeneous wireless networks," IEEE Transactions on Mobile Computing, vol. 11, no. 12, pp. 2073-2086, 2012.

[29] Z. Liu, L. Yu, Y. Gao, S. Hu, and D. Samb, "A constructive capacity lower bound of the inhomogeneous wireless networks." Wireless personal communications, vol. 71, no. 3, 2013.

[30] C. Wang, C. Jiang, Y. Liu, X.-Y. Li, and S. Tang, "Aggregation capacity of wireless sensor networks: Extended network case," IEEE Transactions on Computers, vol. 63, no. 6, pp. 1351-1364, 2014.

[31] A. Keshavarz-Haddad and R. Riedi, "Bounds for the capacity of wireless multihop networks imposed by topology and demand," in MobiHoc, 2007.

[32] R. Zheng, "Asymptotic bounds of information dissemination in powerconstrained wireless networks," IEEE Transactions on Wireless Coтmunications, vol. 7, no. 1, pp. 251-259, 2008.

[33] A. Keshavarz-Haddad and R. Riedi, "On the broadcast capacity of multihop wireless networks: Interplay of power, density and interference," in SECON, 2007.

[34] X. Y. Li, S. Tang, and X. Mao, "Capacity bounds for large scale wireless ad hoc networks under Gaussian channel model," in SECON, 2009.

[35] A. Ozgur, O. Leveque, and D. Tse, "Hierarchical cooperation achieves linear capacity scaling in ad hoc networks," in INFOCOM, 2007.

[36] M. Franceschetti, M. D. Migliore, and P. Minero, "The capacity of wireless networks: Information-theoretic and physical limits," IEEE Transactions on Information Theory, vol. 55, no. 8, pp. 3413-3424, 2009.
[37] M. Ji, Z. Wang, H. R. Sadjadpour, and J. J. Garcia-Luna-Aceves, "The capacity of ad hoc networks with heterogeneous traffic using cooperation," in INFOCOM, 2010

[38] R. Kolte, A. Özgür, and A. El Gamal, "Capacity approximations for Gaussian relay networks," IEEE Transactions on Information Theory, vol. 61, no. 9, pp. 4721-4734, 2015.

[39] O. Dousse and P. Thiran, "Connectivity vs capacity in dense ad hoc networks," in INFOCOM, 2004.

[40] O. Arpacioglu and Z. J. Haas, "On the scalability and capacity of wireless networks with omnidirectional antennas," in IPSN, 2004.

[41] M. Brazil, R. L. Graham, D. A. Thomas, and M. Zachariasen, "On the history of the euclidean steiner tree problem," Archive for history of exact sciences, vol. 68, no. 3, pp. 327-354, 2014.

[42] S. Ramanathan, "A unified framework and algorithm for (T/F/C)DMA channel assignment in wireless networks," in INFOCOM, 1997.

[43] M. Franceschetti, M. Migliore, P. Minero, and F. Schettino, "The degrees of freedom of wireless networksvia cut-set integrals," IEEE Transactions on Information Theory, vol. 57, no. 5, pp. 3067-3079, 2011.

[44] S. Kamath, S. Kannan, and P. Viswanath, "Network capacity under traffic symmetry: Wireline and wireless networks," IEEE Transactions on Information Theory, vol. 60, no. 9, pp. 5457-5469, 2014.

[45] E. Perevalov, R. Blum, and D. Safi, "On the capacity of ad hoc networks with clustering," in IEEE Wireless Communications and Networking Conference, 2005.

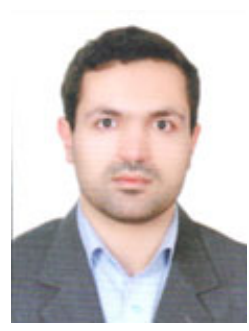

Alireza Keshavarz-Haddad received his B.Eng. degree in 2001 from the Department of Electrical Engineering of Sharif University, Tehran, Iran, and his M.S. and Ph.D. degrees in 2003 and 2007 from the Department Electrical and Computer Engineering of Rice University, Houston, Texas. Since 2008, he has been with the School of Electrical and Computer Engineering at Shiraz University, Shiraz, Iran, where he is currently an Assistant Professor. His research interests are in Wireless Ad Hoc and Sensor networks, Network Coding, and Network Security.

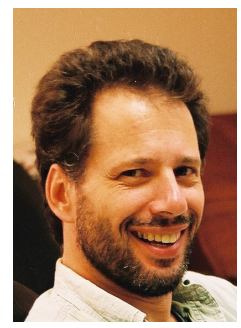

Rudolf H. Riedi received his $\mathrm{PhD}$ in Mathematics in 1993 from the ETH Zürich, Switzerland. He spent 1993-1995 at Yale's Mathematics Department working with Benoit Mandelbrot, 1995-1997 at INRIA Paris, and 1997-2007 at the Brown School of Engineering at Rice University, Houston, where he last held the position of an Associate Professor of Statistics with tenure. Since 2007 he holds the position of a Professeur of Mathematics, associated with the Department of Informatics and Telecommunication at the Haut Ecole of Engineering and Architecture Fribourg, HES-SO University of Applied Sciences Western Switzerland. His research interests lie in multiscale statistics and stochastics with applications in telecommunication and computational finance. 